\title{
BLACK HOLES AND THE PHASES OF BRANE THERMODYNAMICS
}

\author{
Emil J. Martinec \\ Enrico Fermi Institute and Department of Physics \\ University of Chicago \\ 5640 S. Ellis Ave. \\ Chicago, IL 60637-1433 \\ e-martinec@uchicago.edu
}

\section{INTRODUCTION}

Thermodynamics is a useful tool in analyzing the characteristic properties of a system of many degrees of freedom. The equation of state gives the density of states, as well as clues to the effective degrees of freedom and their interactions, at the characteristic energy scale set by the temperature. These lectures will try to convey the idea that brane thermodynamics is a useful way of organizing much of what we know about the duality between quantum gravity and nongravitational theories (often gauge field theories) which generalizes the AdS/CFT correspondence (see [1] for a review).

We will restrict our considerations to systems with maximal supersymmetry compactified on tori; this seemingly simple arena already encompasses a rich variety of phenomena. In section two, the relation between brane dynamics and black holes is reviewed, beginning with the correspondence principle [2] governing the transition between black holes and objects in perturbative string theory. Brane subsystems are isolated by the (Maldacena) scaling limit [3] that decouples their dynamics from the ambient spacetime; the UV/IR relation [4] between energy scales in the brane and gravity descriptions is illustrated in the context of the thermodynamics. Criteria are established governing the validity of various effective descriptions of the thermodynamics of the system. In sections three and four, the application of these criteria are illustrated via a set of increasingly sophisticated examples. Section five concludes with a few closing remarks. 
More details may be found in [5][6][7][8]; these articles were written from the perspective of matrix theory [9] (see [10] for a review), which arises as the universal low-entropy phase of Dp-brane dynamics on tori. The present lectures adopt a somewhat more mainstream viewpoint, adhering more closely to a description in terms of the gauge theory dynamics appearing at high entropy. The phase structure at high entropy, insensitive to the spatial boundary conditions, was first studied in [11], [12].

\section{BRANES AND BLACK HOLES}

At weak coupling, the dynamics of $N$ Dp-branes in type II string theory is governed by open string perturbation theory. The generic state is a gas of open strings; the low-energy modes are those of $p+1$ -

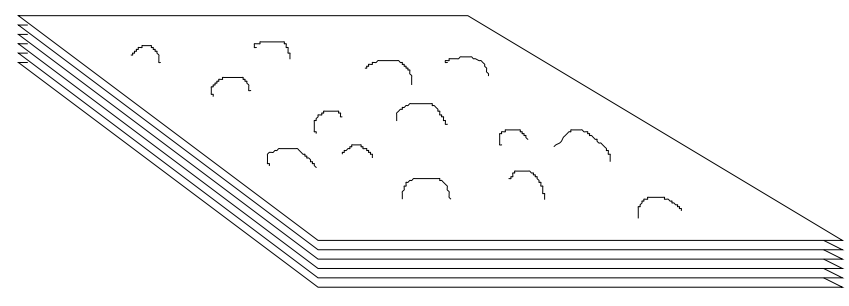

Figure 1.1 Gas of open strings on a stack of D-branes.

dimensional $U(N)$ supersymmetric Yang-Mills theory (SYM). The lowenergy effective action is

$$
\mathcal{S}_{\mathrm{eff}}^{\text {open }}=\frac{1}{g_{\mathrm{YM}}^{2}} \int d^{p+1} y \operatorname{Tr}\left[F^{2}+D X^{I} \cdot D X^{I}-\left[X^{I}, X^{J}\right]^{2}+\text { fermions }\right],
$$

where

$$
g_{\mathrm{YM}}^{2}=g_{s} \ell_{s}^{p-3}
$$

is the Yang-Mills coupling on the branes. For temperatures $T<\ell_{s}^{-1}$ and weak coupling, the open string gas in a periodic box of size $\Sigma$ (coordinate identification $y \sim y+\Sigma$ in all spatial directions along the branes) obeys the equation of state of a perturbative relativistic gas

$$
\begin{aligned}
& S \sim N^{2} \Sigma^{p} T^{p} \\
& E \sim N^{2} \Sigma^{p} T^{p+1} \sim S^{\frac{p+1}{p}}\left(N^{2} \Sigma^{p}\right)^{-1 / p} .
\end{aligned}
$$


On the other hand, Dp-branes are a source of the gravitational and other fields of the closed string sector, whose low energy effective action is

$\mathcal{S}_{\text {eff }}^{\text {closed }}=\frac{1}{G_{10}} \int d^{10} x\left[e^{-2 \phi}\left(\mathcal{R}+4(\nabla \phi)^{2}-\frac{1}{12} H_{(3)}^{2}\right)-\frac{1}{2(p+2) !} \mathcal{F}_{(p+2)}^{2}+\ldots\right]$,

where $\mathcal{F}_{(p+2)}$ is the RR field strength sourced by the Dp-branes, and $G_{10} \sim g_{s}^{2} \ell_{s}^{8}$. The generic state in the superselection sector with $N$ units of RR flux and energy density concentrated at the source is the corresponding black Dp-brane geometry. The solution of the effective field

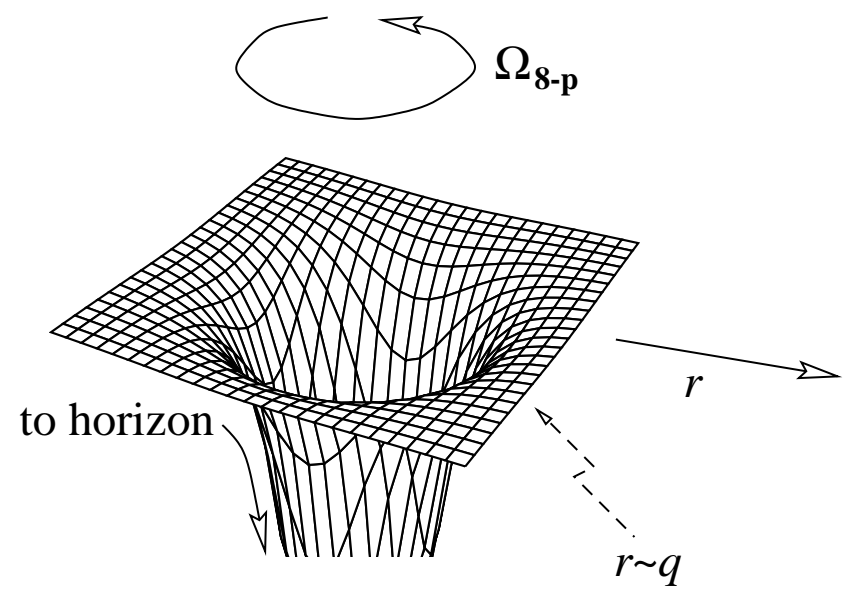

Figure 1.2 Closed string geometry related to the excited D-branes of Figure 1.1, in the spatial directions transverse to the branes.

equations, which applies when the source is macroscopic (i.e. $N$ is sufficiently large), is (see [1],[13] for reviews)

$$
\begin{aligned}
F_{r t y_{1} \ldots y_{p}} & =\partial_{r} H^{-1} \\
e^{\phi} & =g_{s} H^{\frac{3-p}{4}} \\
d s^{2} & =H^{-1 / 2}\left(-h d t^{2}+d \vec{y}_{(p)}^{2}\right)+H^{1 / 2}\left(h^{-1} d r^{2}+r^{2} d \Omega_{8-p}^{2}\right) \\
H & =1+\left(\frac{q}{r}\right)^{7-p}, \quad h=1-\left(\frac{r_{0}}{r}\right)^{7-p}
\end{aligned}
$$

where again the coordinate identification of the directions along the brane is $y \sim y+\Sigma$.

We might expect to compare the open string gas to closed string geometry when the system is not too far from extremality, that is to 
say not very highly excited above the (BPS saturated, supersymmetric) ground state carrying the given charge:

$$
E \ll M_{B P S} \Rightarrow r_{0} \ll q ;
$$

then one has

$$
\begin{aligned}
& \left(\frac{q}{\ell_{s}}\right)^{7-p} \sim g_{s} N \\
& \left(\frac{r_{0}}{\ell_{s}}\right)^{9-p} \sim \frac{S^{2}}{N} g_{s}^{3}\left(\frac{\Sigma}{\ell_{s}}\right)^{-p} .
\end{aligned}
$$

The first relation is simply Gauss' law for the $(p+1)$-form RR flux; the second is the usual Bekenstein-Hawking entropy, determined from the black hole geometry as the area in Planck units of the horizon at $r=r_{0}$. One can read off the ADM energy of the system from the asymptotic metric component $g_{t t} \sim-1+\left(G_{D} M / r^{7-p}\right)$ in the Einstein frame

$$
\ell_{s} E \sim\left[\left(\frac{S^{2}}{N}\right)^{7-p}\left(\frac{\Sigma}{\ell_{s}}\right)^{p(p-5)} g_{s}^{3-p}\right]^{\frac{1}{9-p}} .
$$

The black geometry has lower free energy than the open string gas (1.3) when (note that $\mathcal{F} \sim E \sim T S$ in terms of scaling properties)

$$
\left[g_{s}\left(\frac{\ell_{s}}{\Sigma}\right)^{p-3}\right]^{p}=\left[g_{\mathrm{YM}}^{2} \Sigma^{3-p}\right]^{p}>S^{3-p} N^{p-6} .
$$

In other words, for large enough coupling $g_{\mathrm{YM}}^{2}$, the black geometry takes over. Defining the effective coupling at the scale of the temperature $T \sim E / S$

$$
g_{\mathrm{eff}}^{2}=g_{\mathrm{YM}}^{2} N T^{p-3},
$$

the condition (1.10) means that black geometry is valid for $g_{\text {eff }} \gg 1$, and the open string gas is a valid description for $g_{\text {eff }} \ll 1$; an equivalent statement is that the geometrical description holds when the typical radius of curvature of the geometry at the horizon satisfies $R_{\text {hor }}>\ell_{s}$. This transition between an ensemble of perturbative strings and a black

${ }^{1}$ The Einstein frame is characterized by the field redefinition $\tilde{g}_{\mu \nu}=\exp [\alpha \phi] g_{\mu \nu}$ of (1.4) that makes the gravitational term in the effective action $G_{D}^{-1} \int \sqrt{\tilde{g}} \mathcal{R}[\tilde{g}]$ in the $D=10-p$ non-compact spacetime dimensions transverse to the brane.

${ }^{2}$ One can, for example, use the proper radius (1.5) of angular spheres transverse to the branes. It is straightforward to check that this is string scale at the point $g_{\text {eff }} \sim 1$. 
geometry is an example of the correspondence principle of HorowitzPolchinski [2], which states that a black hole becomes a perturbative string state at sufficiently weak coupling.

One can sharpen the picture considerably by scaling away the asymptotically flat region, following Maldacena [3]:

$$
\ell_{s} \rightarrow 0 \text { with } g_{\mathrm{YM}}^{2} \Sigma^{3-p}, \Sigma E \text { held fixed. }
$$

This limit decouples string oscillator modes, whose energy typically scales as $1 / \ell_{s}$. Since the ten-dimensional gravitational coupling is $G_{10} \sim$ $g_{s}^{2} \ell_{s}^{8}$, while the Yang-Mills coupling on the branes is $g_{\mathrm{YM}}^{2}=g_{s} \ell_{s}^{p-3}$, the closed string dynamics away from the brane source decouples (i.e. $\left.G_{10} \rightarrow 0\right)$ for $p<7\left(G_{10} \rightarrow\right.$ const. for $\left.p=7\right)$. One also encounters situations described by M-theory; here, the relevant gravitational coupling is the eleven-dimensional Newton constant $G_{11} \sim g_{s}^{3} \ell_{s}^{9}$, which scales away for $p<6$ and approaches a constant for $p=6$, in the limit (1.12). Even though the dynamics becomes trivial far from the branes, one presumes that close enough to the source, the scaling away of the gravitational coupling is compensated by large field strengths. Maldacena conjectured that in a region of overlap, quantum gravity is defined by the strongly coupled dynamics of branes; conversely, geometry gives an effective description of the brane dynamics.

An interesting feature of this relation between geometry and brane dynamics is a correspondence between increasing distance from the horizon in the geometry, and high energies in the brane dynamics - the $U V / I R$ correspondence [4][14]. This property is readily seen in the thermodynamics, where the Hawking temperature $T \sim E / S$ of the black geometry is

$$
T \sim\left(\frac{r_{0}}{\ell_{s}^{2}}\right)^{\frac{5-p}{2}}\left(g_{\mathrm{YM}}^{2} N\right)^{-1 / 2} .
$$

Higher temperature (the UV of the brane dynamics) is associated to larger horizon radius (the IR in gravity), up to $p=5.3$ The UV/IR correspondence is related to the positive specific heat of Dp-brane black holes up to $p=5$. The well-controlled UV structure of the brane dynamics then provides an IR regulator for gravity (it implicitly prescribes an asymptotic boundary condition on the geometry), while the Wilsonian renormalization group guarantees a well-behaved UV structure for gravity. This proposal begins to break down for $p=5$, where the brane dynamics exhibits a limiting temperature $T \sim\left(g_{\mathrm{YM}}^{2} N\right)^{-1 / 2}$ known as

${ }^{3}$ Note that this is the same as the UV/IR relation obtained in [14] from the scaling of the wave equation in the near-horizon geometry. 
the Hagedorn temperature. Such behavior is characteristic of string dynamics, and so it has been proposed [15][16][17] that there is a (strongly coupled) string theory decoupled from gravity which governs the dynamics of fivebranes. Going a bit further, for $p=6$ the system has negative specific heat - bulk gravity is not decoupled, as we saw above; there is no IR regulator, and arbitrarily many light states couple at longer and longer wavelengths when we pump energy in to increase the horizon size.

To summarize, in regions of strong effective coupling, the thermodynamics of maximally supersymmetric Yang-Mills theory is described by/describes black geometries. The thermodynamics of black geometry is entirely governed by the geometry at the horizon; thus we can try to map out a phase diagram using whatever low-energy description is appropriate for the horizon geometry in a given strongly-coupled regime, and matching onto perturbative domains at correspondence points.

To implement this strategy, we should examine the constraints on the validity of a low-energy geometrical description:

(1) The curvature at the horizon should be less than the string scale. This is the Horowitz-Polchinski correspondence principle. If this condition is not satisfied, the appropriate description is a gas of perturbative strings (in the presence of the background D-branes).

(2) The string coupling at the horizon should be less than unity. If this condition is not satisfied, then one either

(a) lifts to M-theory in a IIA string description; this repackages the string metric, dilaton, and RR one-form into an elevendimensional metric:

$$
d s_{11}^{2}=e^{-2 \phi / 3} d s_{10}^{2}+e^{4 \phi / 3}\left(d x_{11}+C_{\mu} d x^{\mu}\right)^{2} .
$$

D0-branes carry momentum in the eleventh dimension; strings are membranes wrapped around $x_{11}$; and so on.

(b) performs an S-duality transformation in a IIB description:

$$
\phi \rightarrow-\phi \quad, \quad g_{s} \rightarrow 1 / g_{s} \quad, \quad \ell_{s}^{2} \rightarrow g_{s} \ell_{s}^{2} \quad\left(G_{10} \rightarrow G_{10}\right) .
$$

In particular, perturbative strings are interchanged with D1branes, and D3-branes are invariant due to the S-duality symmetry of $3+1 \mathcal{N}=4$ SYM.

(3) Cycle sizes should be greater than the string scale:

$$
\Sigma_{i} \sqrt{G_{i i}\left(r_{0}\right)}>\ell_{s}
$$


If this condition is not satisfied, one should T-dualize on the corresponding cycles

$$
\begin{aligned}
\Sigma_{i} & \rightarrow \ell_{s}^{2} / \Sigma_{i} \\
\phi & \rightarrow \phi-\frac{1}{2} \sum_{i=1}^{n} \log \left[G_{i i}\right] \quad, \quad g_{s} \rightarrow g_{s} \prod_{i=1}^{n} \frac{\ell_{s}}{\Sigma_{i}} \\
G_{i i} & \rightarrow 1 / G_{i i},
\end{aligned}
$$

where the sums and products are over all affected cycles, and we have assumed a rectangular torus. T-duality [18] exchanges momenta on the affected cycles with perturbative string winding, and mixes the various Dp-branes [19][20]. For example, Dp-branes wrapping $T^{p}$ are exchanged with D0-branes on the dual torus $\tilde{T}^{p}$.

(4) After T-duality, one can have a lower-dimensional brane on a higher-dimensional torus. If the horizon size is larger than the proper size of a particular cycle

$$
\Sigma_{i} \sqrt{G_{i i}\left(r_{0}\right)}<r_{0}
$$

the black hole fills that compact dimension; if not, it is entropically favorable for the horizon to localize on that cycle (see Figure 1.3). This instability to topology change of the horizon was first studied by Gregory and Laflamme [21].
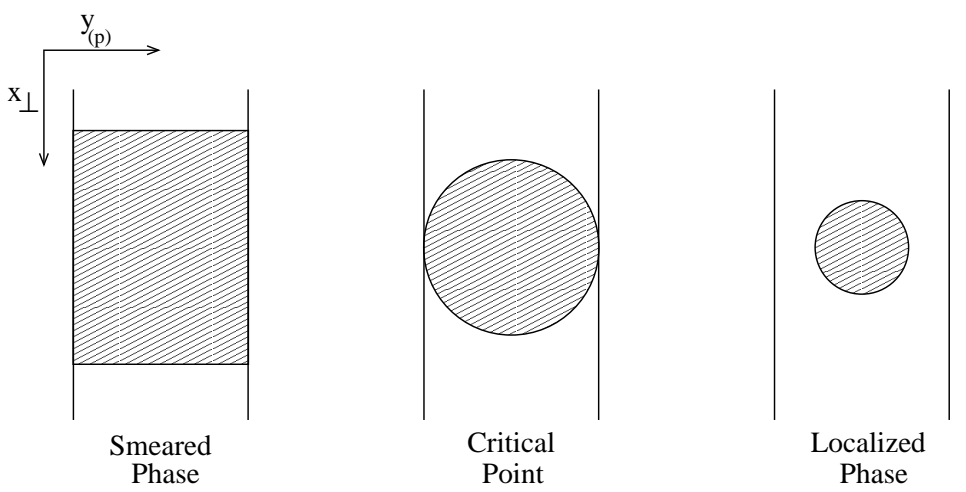

Figure 1.3 Gregory-Laflamme localization transition

(5) A similar phenomenon occurs in the eleventh dimension, for D0brane black holes (see Figure 1.4). After lifting to M-theory, D0brane charge is $p_{11}=N / R_{11}$. Since

$$
G_{11,11}=\exp [4 \phi / 3] \rightarrow \infty \text { as } r_{0} \rightarrow 0,
$$


as the horizon radius decreases, at some point the horizon localizes on the M-circle parametrized by $x_{11}$. The harmonic functions $H, h$
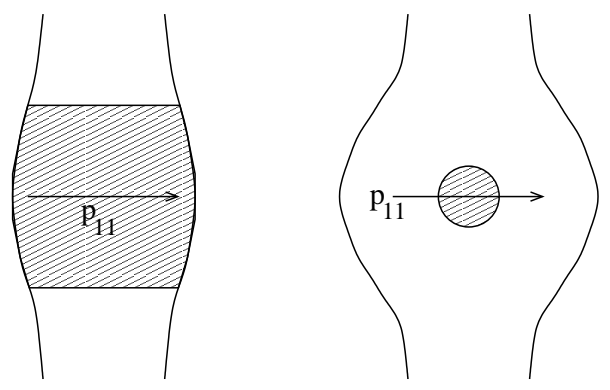

Figure 1.4 Gregory-Laflamme localization transition for a boosted horizon

in the metric pass from being independent of $x_{11}$ to being periodic in $x_{11}$.

To the level of approximation considered here, all equations of state are scaling relations of the form

$$
\Sigma E \sim \text { const. } \times S^{a} N^{b} V^{-3 c}
$$

where

$$
V^{-3} \equiv g_{\mathrm{YM}}^{2} \Sigma^{3-p}
$$

is the effective coupling. 6 Furthermore, the free energy $\mathcal{F}$, energy $E$, and $T S$ all scale the same way. Thus, to find the boundaries between different phases, we may equate the dimensionless energies $(\Sigma E)_{\mathrm{phase} 1}=$ $(\Sigma E)_{\text {phase } 2}$; then the phase boundary occurs at

$$
\frac{\log S}{\log N}=\alpha \frac{\log V}{\log N}
$$

in the large $\mathrm{N}$ limit. Consequently, it is convenient to plot the phase diagram as a function of $\left(\frac{\log S}{\log N}\right)$ versus $\left(\frac{\log V}{\log N}\right)$.

Some remarks are appropriate at this point on what is meant in these lectures by the terms 'phase' and 'phase transition'. For the present purpose, we distinguish thermodynamic phases by the scaling of their equations of state. At finite brane charge $N$ and in finite volume, there

\footnotetext{
${ }^{4}$ The choice of $V$ as the inverse one-third power of the dimensionless gauge coupling is motivated by the fact that $V$ is the size of the dual torus $\tilde{T}^{p}$ (in $11 \mathrm{~d}$ Planck units) seen by D0-branes in low-entropy phases.
} 
cannot be any true phase transitions, since the number of available degrees of freedom will be finite; rather one has a crossover between limiting behaviors. In some cases, the transition is a true phase transition in the classical limit $N \rightarrow \infty$. An example is the Gregory-Laflamme transition discussed above; in [21] it was found that the classical equations of motion develop an unstable mode as one enters the regime (1.18), the hallmark of a second-order phase transition. In other examples, such as the Horowitz-Polchinski correspondence transition, it is not known whether the classical limit develops a singularity; classical black holes might or might not smoothly evolve into perturbative classical string states. The dynamics of such transitions has been studied in [22][23]; the details are dimension-dependent.

\section{DP-BRANE EXAMPLES \\ 3.1 WARM-UP EXERCISE: DO-BRANES}

Consider first the example of D0-branes. Here there is no compactification scale $\Sigma$ since all spatial dimensions are noncompact (apart from the M-circle). The Yang-Mills coupling $g_{\mathrm{YM}}$ sets the only scale; and the phase plot is one-dimensional, since phase boundaries are set by a condition of the form $\left(\frac{\log S}{\log N}\right)=\alpha$. From (1.10), the Horowitz-Polchinski correspondence point for $p=0$ is $S \sim N^{2}$, at which $r_{0} / \ell_{\mathrm{pl}} \sim N^{1 / 3}$. For $S>N^{2}$, the horizon radius in Planck units is larger than $N^{1 / 3}$; and from (1.13) we see that the Hawking temperature is more than the energy $r_{0} / \ell_{s}^{2}$ required to stretch a string across the horizon scale $r_{0}$, indicating that the D0-brane quantum mechanics is deconfined. On the other hand, for $S<N^{2}$ the thermodynamics of the ensemble of D0-branes is well-described by the black D0 geometry. Strong string coupling $\left.e^{\phi}\right|_{\text {hor }} \sim 1$ sets in at $S \sim N^{8 / 7}$, as one sees from (1.5),(1.8). For lower entropies/energies, the proper effective description of the horizon geometry (and therefore the thermodynamics) takes place in eleven dimensional M-theory. Finally, horizon localization on the M-circle $x_{11}$

\footnotetext{
${ }^{5}$ Here and below, unless otherwise stated, the Planck scale will always refer to that of elevendimensional M-theory, $G_{11} \sim \ell_{\mathrm{pl}}^{9} \sim g_{s}^{3} \ell_{s}^{9}$.
} 
occurs for entropies below $S \sim N$. We can combine all these considerations into the phase diagram shown in Figure 1.5.

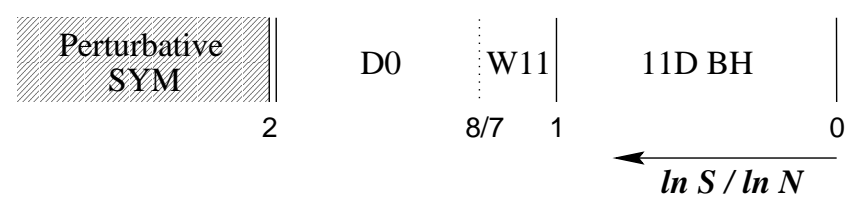

Figure 1.5 D0-brane phase diagram.

\subsection{DP-BRANES ON $T^{P}$ FOR $P>0$}

Dp-brane thermodynamics for $p>0$ has a phase structure that depends on both $\log S / \log N$ and the dimensionless Yang-Mills coupling $\log V / \log N$. At sufficiently weak coupling (large $\log V / \log N)$, the phase structure for all $p$ is essentially the same as for D0-branes at low enough entropy, since the dynamics must eventually settle into the quantum mechanics of the momentum zero-modes on the torus. Conversely, at high entropy (high temperature), the finite volume effects are irrelevant; one should see a correspondence transition as the coupling is increased (smaller $V$ ). The correspondence transition is thus bounded by Equation (1.10) or $S \sim N^{2}$, whichever is met first coming from weak coupling. Figure 1.6 illustrates what happens as the dimension increases. The shaded region denotes the perturbative gas phase; the double line is the correspondence transition curve. The horizontal part of the correspondence boundary separates the perturbative gas from the black D0-brane phase discussed above. The angled continuation of the correspondence curve delineates the transition to the black Dp-brane phase. The slope of this part of the correspondence curve separating the phase of black Dp-branes from the perturbative gas phase changes with $p$. The direction of the change is in accord with the scaling of the effective coupling with energy (temperature): For $p<3$, one passes from the perturbative phase to the strong coupling, geometrical phase by de-

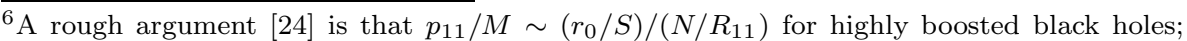
therefore, for $N \sim S$, the 'Lorentz contraction' $p_{11} / M \sim r_{0} / R_{11}$ is such that the horizon is just contained within the M-circle. Closer inspection [25] reveals that the black hole horizon does not Lorentz contract, being a surface of infinite redshift; rather, the metric backreacts on the momentum stress-energy of the black hole, expanding by exactly the same factor $p_{11} / M$ (hence the bulge depicted in Figure 1.4). Thus the net effect is the same: The phase boundary of horizon localization along the M-circle always occurs at $N \sim S$.
} 


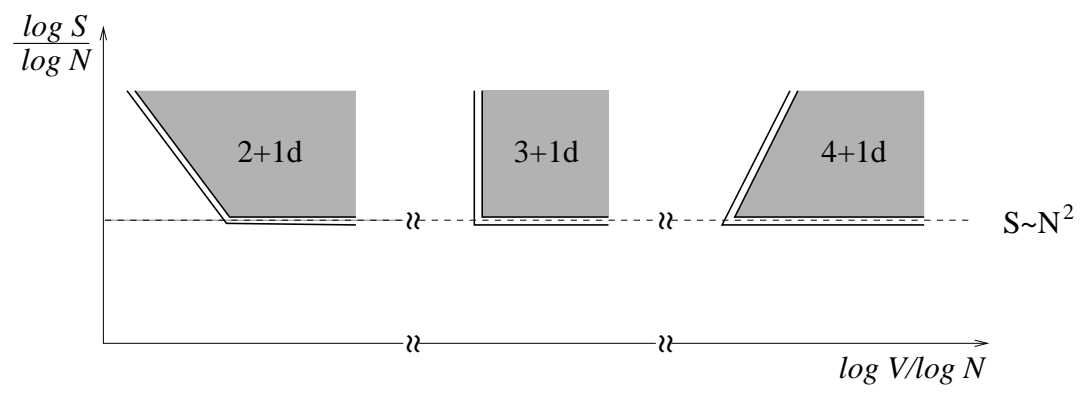

Figure 1.6 Correspondence transition for various $p$.

creasing the temperature/entropy; $p=3$ is marginal; and for $p>3$, strong coupling is met upon increasing the temperature/entropy.

One expects that the freezing of the brane dynamics into the quantum thermodynamics of its zero-modes should persist at strong coupling. The strong coupling thermodynamics is coded in the geometry of the horizon; the transition to a zero-mode description is seen as the necessity to perform a T-duality transformation of the horizon geometry in order to maintain a valid effective description, according to criterion (3) of section 2.7 On the geometrical side, this is seen from the proper size of the torus $T^{p}$ wrapped by the Dp-branes as the horizon radius decreases. Below some value of $r$, the T-dual description, for which the size of $\tilde{T}^{p}$ increases with decreasing $r$, is the valid one; see Figure 1.7. Let us make this change of description; we are now in a D0-brane geometry at the horizon, smeared over the dual torus $\tilde{T}^{p}$. As we continue to lower the entropy, the horizon radius decreases, so eventually we should violate the criterion (4), and the horizon localizes on the dual torus. Also the dilaton runs toward strong coupling at small radius in a D0-brane background (Equation (1.5)); at some point we will also have to pass to an M-theory description.

3.2.1 D3-branes. Putting together all these considerations leads to a phase diagram for $V>1$. This is illustrated in Figure 1.8 for the D3-brane case, where we have extended the structure to $V<1$ using the S-duality symmetry of maximally supersymmetric $3+1 \mathrm{~d}$ superYang-

\footnotetext{
${ }^{7}$ As far as the gauge theory dynamics is concerned, there is no important distinction between the T-dual description in terms of the quantum mechanics of D0-branes, as opposed to the dimensional reduction of the Dp-brane gauge theory, at temperatures sufficiently smaller than $\Sigma^{-1}$.
} 


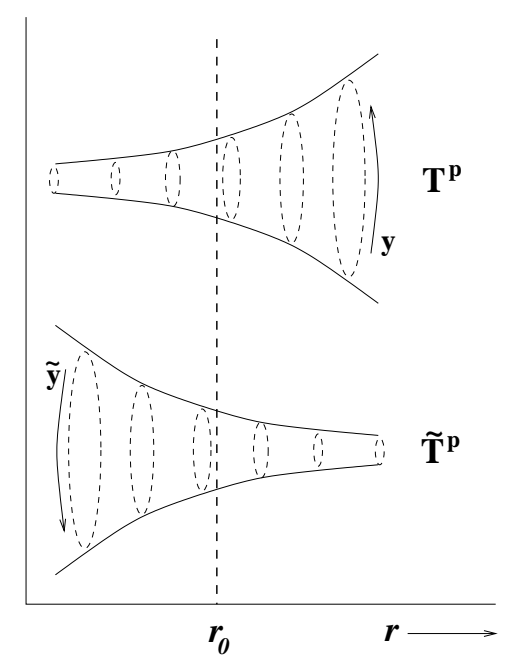

Figure 1.7 Radial dependence of torus proper size, as seen by Dp-branes on $T^{p}$ (top), and D0-branes on $\tilde{T}^{p}$ (bottom).

Mills, which in the present notation sends $V \rightarrow 1 / V$, and reflects all phase structure about the center of the diagram. The following phases occur:

- A: Perturbative D3-brane gas;

- B: Black D3-brane;

- C: Black D0-brane;

- D: Boosted 11d Schwarzschild black hole, localized on $\tilde{T}^{3}$;

- E: Boosted 11d Schwarzschild black hole, smeared on $\tilde{T}^{3}$.

The corresponding regions obtained by S-duality are labelled similarly. Phase boundaries are denoted by solid lines, and labelled by lower case letters:

- a: D3-perturbative gas correspondence transition.

- a': D0-perturbative gas correspondence transition.

- b: Gregory-Laflamme horizon localization transition of the D0brane horizon on the dual torus $\tilde{T}^{3}$. The horizon localized over the compact space is denoted $D 0$, while the horizon smeared over the compact space denoted by an overline $\overline{D 0}$; similarly, $W 11$ and $\overline{W 11}$ are the lifts to M-theory of the respective geometries. 


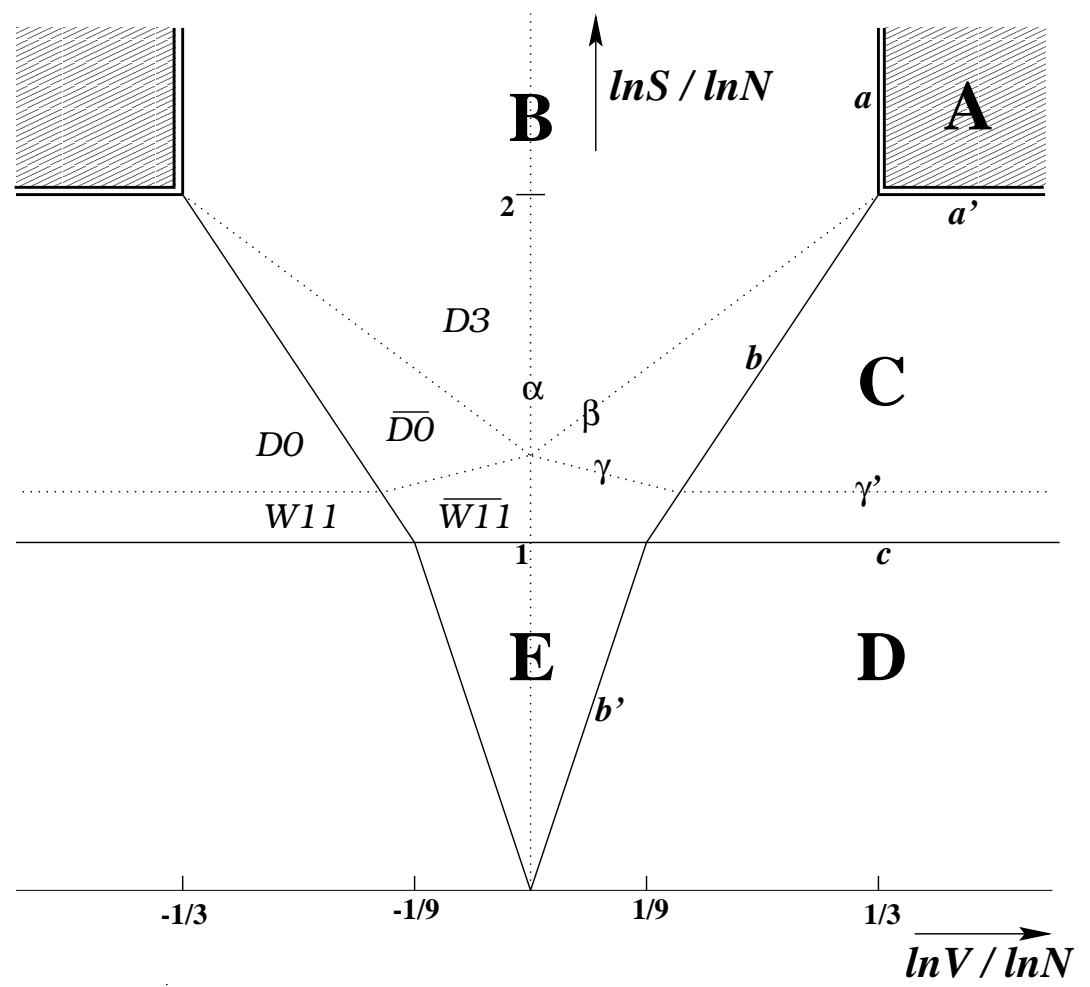

Figure 1.8 Phase diagram for D3-branes on $T^{3}$. The phases describing various regimes are A: Perturbative D3-brane gas; B: Black D3-brane; C: Black D0-brane; D: Boosted 11d Schwarzschild black hole, localized on $\tilde{T}^{3}$; and E: Boosted 11d Schwarzschild black hole, smeared on $\tilde{T}^{3}$. The labels of various patches $(D 0, \bar{D} 0$, $W 11$, etc.) and transition curves $a, b, c ; \alpha, \beta, \gamma$ are explained in the text. The symmetry about the center of the diagram is a reflection of the S-duality symmetry of $3+1 \mathrm{~d} \mathcal{N}=4 \mathrm{SYM}$.

- b': Continuation of the localization transition below $S \sim N$ (the slope changes when the phase boundary $(c)$ is crossed, due to the change in horizon topology).

- c: Localization of the horizon on $x_{11}$ at $S \sim N$.

We distinguish correspondence transitions from localization transitions by denoting the former with double lines, the latter by single lines. Again, to avoid clutter only half of the transition curves are designated, the others being copies under S-duality.

Within a given phase it may be necessary to perform duality transformations, lifting to M-theory, or reduction from M-theory to string the- 
ory, in order to maintain a proper low-energy description of the horizon geometry according to the criteria of Section 2. These transformations are changes of description of the horizon geometry which do not affect the equation of state, thus are not phase boundaries. They are denoted by dotted lines and labelled by greek letters:

- $\alpha$ : S-duality at $g_{\mathrm{YM}}=1$;

- $\beta$ : T-duality when the proper size of $T^{3}$ is string scale;

- $\gamma-\gamma^{\prime}$ : Lift to M-theory when the string coupling becomes order one at the horizon.

The patches where different low-energy descriptions hold within a given phase are indicated on the left half of Figure 1.8.

Note that for $\left(\frac{\log V}{\log N}\right)>1 / 3$ the structure is that of D0-branes, see Figure 1.5. However, there is a slight difference; approaching the correspondence curve from above, the temperature of the perturbative open string gas is $T_{+} \rightarrow \Sigma^{-1}$; approaching from below in the geometrical black D0 brane phase, one has $T_{-} \rightarrow \Sigma^{-1}\left(g_{\mathrm{YM}}^{2} N\right)^{1 / 3}$, which is less than $T_{+}$all the way until the end of the correspondence curve at $V^{-1}=\left(g_{\mathrm{YM}}^{2} N\right)^{1 / 3} \sim 1$. This indicates an intermediate phase, not visible when the phase structure is plotted as a function of entropy. One imagines that as this isentropic phase is traversed, the holonomy of the $\mathrm{U}(\mathrm{N})$ gauge fields on the torus become frozen, since they are fluid for high entropy and frozen [24] for low entropy.

3.2.2 D2-branes and D4-branes. The structure of the phase diagram for $V>1$ is essentially the same for all Dp-branes wrapping $T^{p}$, differing only in the slope of the corresponence curve, Figure 1.6. The analogous phases and transition curves carry the same labels as in Figure 1.8. However, for $p \neq 3$, there is typically no symmetry relating the phase diagram at $V<1$ to that for $V>1$, and further analysis is necessary for this regime. The details depend on $p$, and a case-by-case analysis is necessary. We will illustrate with the examples of D2-branes and D4-branes (see Figures 1.9 and 1.10). Small $V$ corresponds to large coupling in the Dp-brane gauge theory, at the scale of the torus cycle size; thus one can think of the passage to small $V$ at large entropy/temperature (where $p+1$ dimensional kinematics applies) as the strong coupling limit. For $p=2$, this is a flow to the IR; for $p=4$ one flows to the UV.

Maximally supersymmetric Yang-Mills theory in 2+1d flows to a nontrivial fixed point in the IR. On the geometrical side, one sees this in the thermodynamics as a transition of the horizon geometry to a structure 


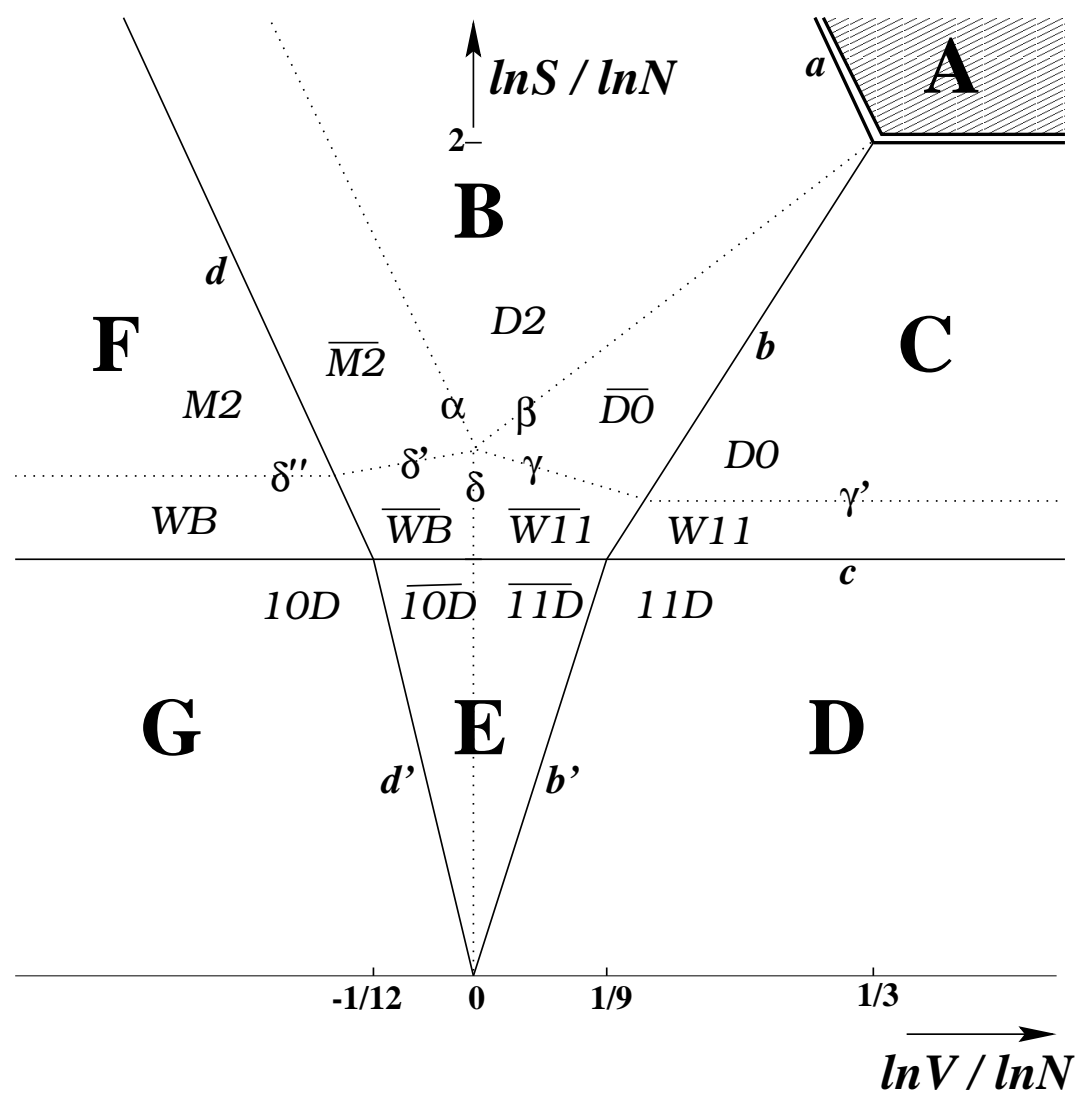

Figure 1.9 D2-brane phase diagram. The phases are - A: Perturbative D2-brane gas. B: Black D2-brane. C: Black D0-brane. D: Boosted 11d Schwarzschild black hole, localized on $\tilde{T}^{2}$. E: Boosted 11d Schwarzschild black hole, smeared on $\tilde{T}^{2}$. F: Black M2-brane. G: Boosted 10d Schwarzshild black hole, localized on $\tilde{S}^{1}$.

that is locally $A d S_{4} \times S^{7}$, with an equation of state

$$
E \sim S^{3 / 2}\left[N^{3 / 2} \Sigma^{2}\right]^{-1 / 2}
$$

The energy-versus-entropy scaling is determined by conformal invariance; the effective number of field theoretic degrees of freedom is the 
coefficient of the spatial volume $V^{p}$ in the last factor, i.e. $N^{3 / 2} \sqrt{8}$ it would be interesting to have an explanation of this scaling from the gauge theory side. The scaling (1.23) differs from that of the D2-brane, Equation (1.9). Starting from the black D2 correspondence curve and continuing to the left at constant entropy, one first lifts to the M-theory black M2-brane geometry when the dilaton at the horizon becomes order one (the dotted line labelled $\alpha$ in the figure); the M2-brane being pointlike in the eleventh dimension, the horizon can localize along the M-circle, and does so along the transition curve $(d)$ in Figure 1.9 (the curve ( $d^{\prime}$ ) is its continuation below $S \sim N$ ). It is this localized M2-brane phase that has the equation of state (1.23).

If we now start in this M2-brane phase, and pass to smaller entropy/temperature at fixed $V$, the size of the $T^{2}$ wrapped by the M2branes (measured at the horizon) shrinks until it becomes Planck size. M-theory on a sub-Planckian two-torus is dual to a circle in IIB string theory [26][27], and the M2-brane stack dualizes into a black wave along this circle. This change of description is the dotted line $\left(\delta-\delta^{\prime}-\delta^{\prime \prime}\right)$ in the figure. Eventually the wave localizes along this circle to a boosted black hole in IIB string theory. All told, the set of phases is
A: Perturbative D2-brane gas.
B: Black D2-brane.
C: Black D0-brane.
D: Boosted 11d Schwarzschild black hole, localized on $\tilde{T}^{2}$.
E: Boosted 11d Schwarzschild black hole, smeared on $\tilde{T}^{2}$.
F: Black M2-brane.
G: Boosted 10d Schwarzshild black hole, localized on $\tilde{S}^{1}$.

Phases A-E are analogues of those appearing in the D3-brane case; the strong-coupling phases $\mathbf{F}$ and $\mathbf{G}$ are new.

Maximally supersymmetric Yang-Mills in $4+1 \mathrm{~d}$ flows to strong coupling in the UV, and thus one must find the strong coupling fixed point which it descends from in order that the theory is well-defined. Geometry again supplies the answer [28][29][11]: The D4-brane geometry lifts to M-theory five-brane at strong coupling, and the required fixed point is a six-dimensional nonabelian tensor field theory with $(2,0)$ supersymmetry, about which little is known. From (1.9), the equation of state of

${ }^{8}$ On the other hand, D3-branes have $N^{2}$ degrees of freedom - one can track them from weak coupling (1.3) to strong coupling (1.9) along a marginal line. 
this six-dimensional theory is

$$
E \sim S^{6 / 5}\left[N^{3}\left(g_{\mathrm{YM}}^{2} \Sigma^{4}\right)\right]^{-1 / 5}
$$

and one can check that the horizon geometry of the M-lifted metric (1.5) is locally $A d S^{7} \times S^{4}$. Because the M-circle lies parallel to the brane, in this case there can be no localization transition of the horizon along this direction; hence the D4 equation of state merely needs to be reinterpreted as that of a higher-dimensional field theory. We see that the theory lives on a torus, four of whose coordinates have period $\Sigma$, while the fifth is of size $g_{\mathrm{YM}}^{2}$ [28]; the effective number of degrees of freedom appears to be $N^{3}$ (more about this in section 4 below).

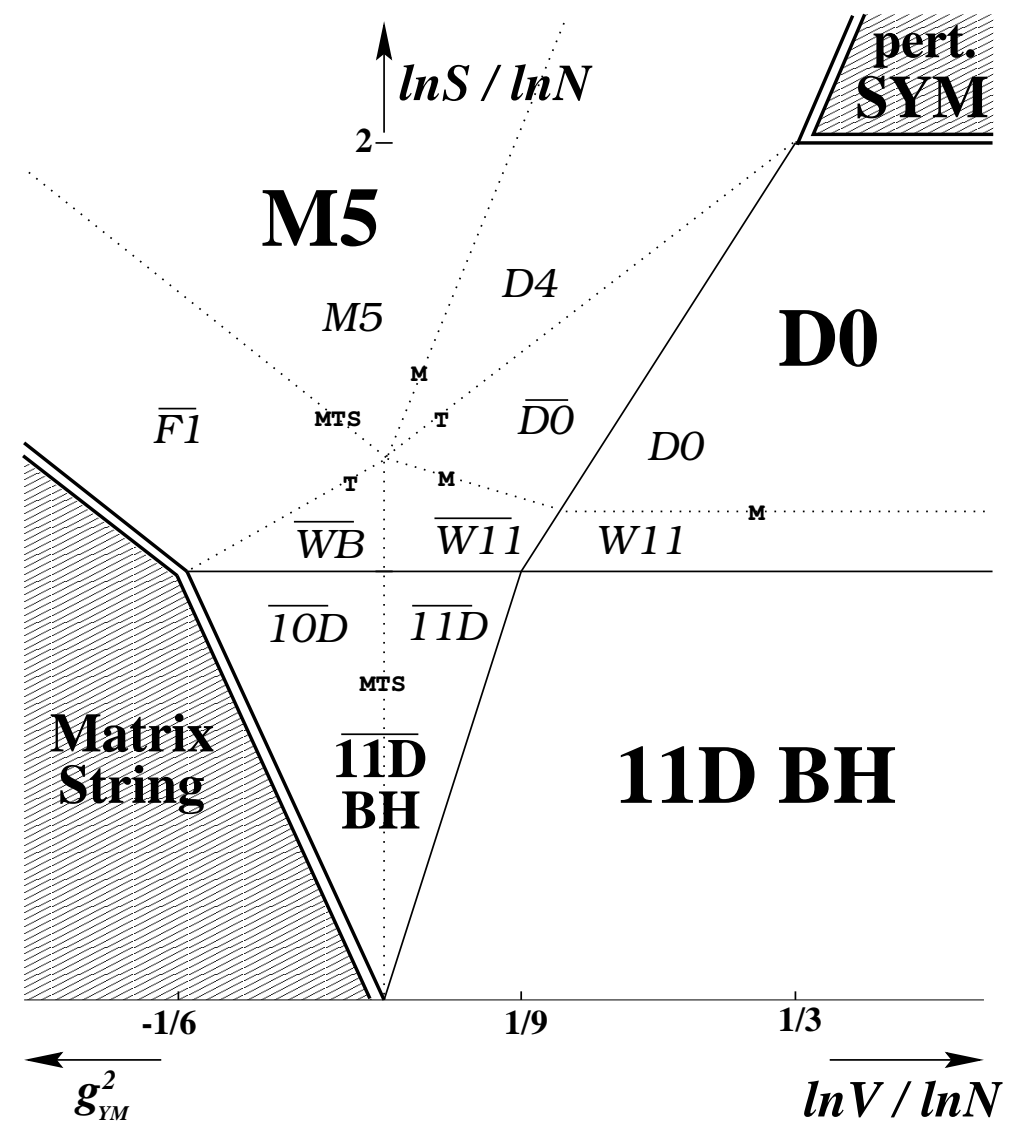

Figure 1.10 D4-brane phase diagram. 
By now the structure of duality transformations/phase transitions should be familiar, so they are labelled directly on Figure 1.10. One new feature - a correspondence transition at strong coupling - does arise at the lower left. As the entropy/temperature is lowered in this regime, The horizon size of the original $T^{4}$ shrinks, while the M-circle remains somewhat larger. Thus at some point we will want to reduce to IIB string theory along the $T^{4}$ This sequence of transformations turns the D4/M5-brane charge into IIB perturbative string winding around the M-circle (the circle related to the $4+1 \mathrm{~d}$ SYM coupling). The resulting perturbative string should have a correspondence transition where a thermally excited, perturbative macroscopic string has less free energy than the black geometry. Thus the phase labelled 'Matrix String' in the figure: A perturbative string arises from the strong coupling dynamics of a gauge theory, as in [30][31][32].

\subsection{QUALITATIVE CHECKS}

The strong-coupling regime of the phase diagram has been evaluated using black geometry. How much of this structure can we motivate from the gauge theory side?

- $S<N$ : One can take the D0-brane interactions determined from the Born-Oppenheimer approximation; the virial theorem and mean field theory then lead to the equation of state scaling of boosted 11d black holes [24][25][33] (in the same spirit as one estimates the size and properties of an atom using the virial theorem and uncertainty principle).

- $N<S<N^{2}$ : Miao Li [34] has given the following estimate of the properties of the localized black D0-brane phase: Suppose the matrix eigenvalue distribution of the D0-brane quantum mechanics is given by

$$
\rho(r)=|\psi(r)|^{2} \propto \frac{N}{L^{9}}\left(\frac{r}{L}\right)^{\alpha},
$$

with $L=N^{1 / 3} \ell_{\mathrm{pl}}$ the scale set by 't Hooft scaling (see the discussion in section 3.1). The scaling exponent $\alpha$ is determined by demanding that the free energy of off-diagonal matrix elements

\footnotetext{
${ }^{9}$ The corresponding dotted line on the figure is labelled MTS, since one way to approach the problem is to select one of the cycles of the $T^{4}$ for reduction to IIA string theory; then one finds that the remaining three are substringy and must be T-dualized, leading to IIB string theory; finally, the resulting string coupling is larger than one, so an S-duality must be performed. At this point all the criteria of section 2 are met.
} 
reproduces the equation of state

$$
E \sim \frac{R_{11}}{\ell_{\mathrm{pl}}^{2}}\left(\frac{S^{2}}{N}\right)^{7 / 9}
$$

in the mean field theory approximation; then $\frac{1}{N}\left\langle\operatorname{tr}\left[X^{2}\right]\right\rangle \sim r_{0}^{2}$ correctly reproduces the horizon scale as a nontrivial self-consistency check.

- $S \sim N^{2}$ (from below): as discussed in section 3.1, at this point the Hawking temperature matches the energy required to excite the off-diagonal matrix elements of the D0-brane quantum mechanics, which are the stretched strings between the D0 particles; this indicates that a deconfinement transition is taking place.

- Horizon localization: The Gregory-Laflamme localization transition take place uniformly for all $p$ along a curve $S \sim V^{9 / 2} N^{1 / 2}$. Susskind [35] has argued that this is an analogue of the GrossWitten large $N$ phase transition of pure $1+1 \mathrm{~d}$ gauge theory on a circle. In the Gross-Witten toy model, the eigenvalues of the gauge field zero-mode (the only gauge-invariant degrees of freedom) undergo a phase transition. The eigenvalues themselves live on a circle, and at high temperature they fill the circle, while at low temperature they clump together. Similarly, the locations of D0-branes on a $p$-torus $\tilde{T}^{p}$ are the eigenvalues of the gauge field on the dual torus $T^{p}$. A smeared horizon has the D0-branes spread across the torus cycles, while a localized horizon clumps the eigenvalues together. The details probably differ from the toy model. For instance, the Gross-Witten transition is third order, whereas the Gregory-Laflamme transition proceeds via the development of a soft mode in the perturbation spectrum of the horizon, leading one to expect a second-order transition.

- Qualitative phase structure. Determined above via geometry, the phase structure matches what is known for the strongly-coupled gauge theory. Maximally supersymmetric Yang-Mills has strongcoupling fixed points for $p=1,2,3,4$ [36], and we have seen in the examples that the appropriate regime of the phase diagram has the equation of state scaling in the manner dictated by conformal invariance.

One of the intriguing issues is why such obviously naive arguments seem to be so successful in capturing the properties of the strong coupling, geometrical phases; for instance, why does mean field theory yield the correct scaling exponents? 


\subsection{PHASES OF $3+1 \mathrm{D}$ SYM ON $S^{3}$}

The low-entropy/temperature structure of brane dynamics on a torus depends heavily on the global topology; the $E_{p+1}$ U-duality [20] was an essential ingredient in maintaining a valid effective description of the horizon geometry in strong effective coupling. There is no such duality group for gauge theory on the sphere, so we should expect a rather different phase structure. Nevertheless, when geometry describes the thermodynamics, we expect the same sorts of phenomena: correspondence transitions bound the validity of the geometrical description; and localization transitions can occur when the horizon is of the same order as a cycle size, if the brane is not wrapped around that cycle. The scalings of the various transitions were worked out in [37], and the results are plotted in Figure 1.11. The structure at $S>N^{2}$ is the same as for

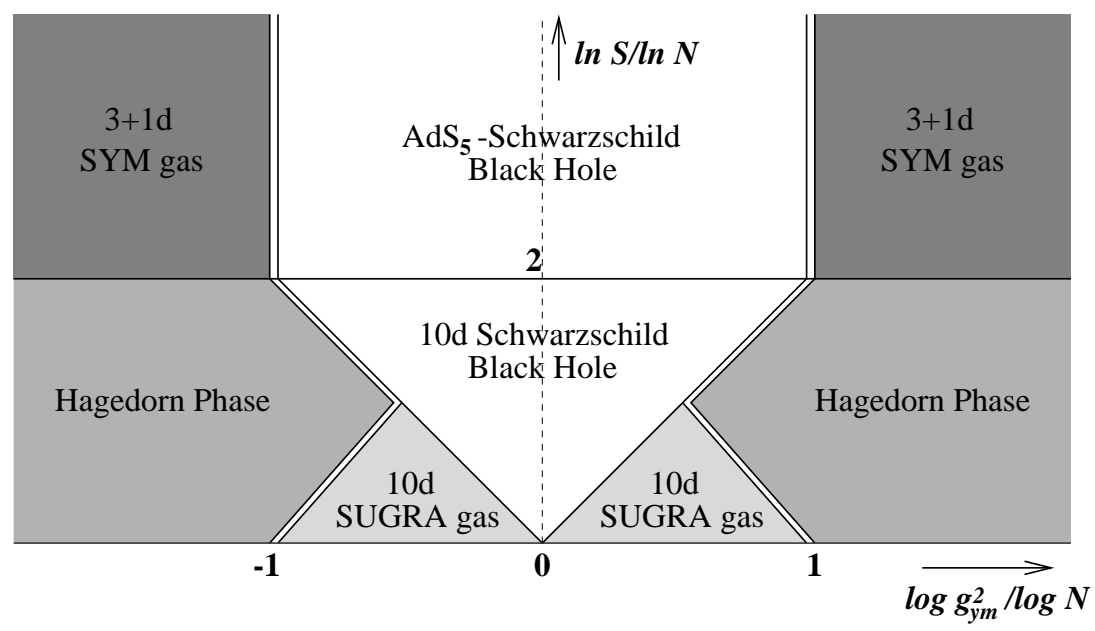

Figure 1.11 Phase diagram of D3-branes on $S^{3}$.

the three-torus, since here the thermal wavelength is smaller than the size of the compact space and the boundary conditions are irrelevant. The perturbative open string gas has a correspondence transition when the 't Hooft coupling (or its dual) is of order one, to an $A d S_{5} \times S^{5}$ Schwarzschild black hole; the horizon size is larger than (and hence smeared across) the five-sphere transverse to the branes. There is a localization transition in this strong-coupling region at $S \sim N^{2}$ (horizon radius of order the five-sphere scale), to a ten-dimensional Schwarzschild black hole completely localized on $A d S_{5} \times S^{5}$. The free energy of a perturbative string (whose radius of gyration is much larger than the radius 
of curvature of the ambient anti-deSitter space) wins out over this black hole at sufficiently weak coupling, leading to a continuation of the correspondence curve below $S \sim N^{2}$. A dilute gas of gravitons in $A d S_{5} \times S^{5}$ dominates at the lowest entropies at strong coupling; the phase transition to a 10d Schwarzschild black hole is just the gravitational Jeans instability. Note that both the Hagedorn phase and the localized 10d black hole phase would be missed in an analysis using the canonical ensemble; since these two phases do not have positive specific heat, coupling the system to a heat bath pumps energy/entropy into the system such that one jumps directly from the supergravity gas phase to the smeared black hole phase above $S \sim N^{2}$.

\section{THE D1-D5 SYSTEM AND PHASES OF 'LITTLE STRING' THEORY}

As a final illustration of the application of thermodynamic techniques to learn about the properties of brane dynamics, consider [7] the D1D5 system compactified on a torus $T^{4} \times S^{1}$, with the four-torus having volume $V_{4}$ and the circle having radius $R$. This system has a rich history (see for example [38]). Here, we will see in the thermodynamics some hints of the origin of the apparently $O\left(N^{3}\right)$ degrees of freedom of the M-theory fivebrane observed in (1.24).

We have seen that, as the entropy is lowered, U-duality transmutes brane charge into momentum; we obtained the infinite momentum frame description of Schwarzschild black holes in M-theory. Adding a second charge to the system, we should expect that we will now find a boosted brane at low entropies. The D1-D5 system is such that we find the IMF description of M-theory fivebranes at low momentum [39]. The associated classical geometry is

$$
\begin{gathered}
d s^{2}=\left(H_{1} H_{5}\right)^{-1 / 2}\left(-h d t^{2}+d y_{5}^{2}\right)+H_{1}^{1 / 2} H_{5}^{-1 / 2}\left(d y_{1}^{2}+\ldots+d y_{4}^{2}\right) \\
+\left(H_{1} H_{5}\right)^{1 / 2}\left(h^{-1} d r^{2}+r^{2} d \Omega_{3}^{2}\right) \\
H_{a}=1+\frac{q_{a}^{2}}{r^{2}} \quad a=1,5 \quad ; \quad h=1-\frac{r_{0}^{2}}{r^{2}},
\end{gathered}
$$

with $y_{i} \approx y_{i}+V_{4}^{1 / 4}, i=1 \ldots 4$, and $y_{5} \approx y_{5}+R$. One can think of this system as a stack of $Q_{5}$ fivebranes in a superselection sector with $Q_{1}$ embedded one-branes. A D1-brane inside a D5-brane is the same as an instanton in the D5 gauge theory [40][41]; since instantons have codimension four, the instanton in $5+1$ superYang-Mills is a $1+1 \mathrm{~d}$ soliton (effectively a string). The instanton moduli space has bosonic dimension of order $Q_{1} Q_{5}$, the number of perturbative 1-5 strings; thus one might 
expect that $k \equiv Q_{1} Q_{5}$ is the measure of the number of degrees of freedom in the system.

Without the D1 charge $Q_{1}$, we saw (Equation (1.13)) that the thermodynamics of $Q_{5}$ D5-branes had a limiting temperature - Hagedorn behavior - characteristic of an underlying string with an (inverse) tension

$$
T_{\max } \sim\left(\alpha_{\mathrm{eff}}^{\prime}\right)^{-1 / 2} \quad, \quad \alpha_{\mathrm{eff}}^{\prime}=g_{\mathrm{YM}}^{2} Q_{5}
$$

(Note that $g_{\mathrm{YM}}^{2}=g_{s}^{2} \ell_{s}^{2}$ here, so that $\left.\alpha_{\text {eff }}^{\prime} \neq \ell_{s}^{2} !\right)$. The limit which decouples the asymptotically flat region is

$$
\ell_{s} \rightarrow 0 \text { with } E \alpha_{\text {eff }}^{\prime 1 / 2}, \quad \frac{R}{\alpha_{\text {eff }}^{\prime 1 / 2}}, \quad \text { and } \frac{V_{4}}{\alpha_{\text {eff }}^{\prime 2}} \text { held fixed. }
$$

Note that this is NOT the limit usually taken $-\ell_{s} \rightarrow 0$, with $E R, g_{s}$, and $V_{4} / \ell_{s}^{4}$ held fixed - which yields the much-studied $A d S_{3} \times S^{3} \times T^{4}$ near-horizon geometry (c.f. A. Strominger's lectures). This latter limit shrinks away the four-torus transverse to the D1 winding, so that the system becomes $1+1$ dimensional. Here we are interested in maintaining the full $5+1$ dimensional nature of the little string dynamics. In terms of the radial scales in the geometry, we are taking $q_{5} \gg q_{1}, r_{0}$; different regimes will arise depending on whether $q_{1}>r_{0}$ or $q_{1}<r_{0}$.

The energy in the system can again be read off the 5d Einstein frame metric, and the entropy is as always the horizon area in (10d) Planck units. After a bit of algebra, one can process the equation of state into the following form: At high entropies, the strongly coupled equation of state is

$$
E=-p_{\|}+\sqrt{\left(S / 2 \pi \alpha_{\mathrm{eff}}^{\prime 1 / 2}\right)^{2}+p_{\|}^{2}},
$$

where $p_{\|}=k R / \alpha_{\text {eff }}^{\prime} \equiv k / \tilde{R}$ (i.e. $\tilde{R}=\alpha_{\text {eff }}^{\prime} / R$ ) behaves as an effective momentum carried by the system - the equation of state is that of a relativistic system of invariant mass $M=S / 2 \pi \alpha_{\text {eff }}^{\prime 1 / 2}$. Thus we have a boosted Hagedorn gas. In the infinite momentum frame limit $p_{\|} \gg M$, we recover the equation of state $S=2 \pi \sqrt{k \cdot 2 R E}$ of BTZ black holes; this is the further limit required to obtain the $A d S_{3}$ horizon geometry.

Since both types of brane are wrapped around the circle of radius $R$, this circle shrinks the fastest with decreasing horizon radius; we should expect to have to T-dualize it relatively early on in applying the criteria of section 2. The resulting D0-D4 system can localize on the dual circle (loosely speaking, the one of radius $\tilde{R}$ ). The localization transition is nothing other than the Hagedorn transition of the effective string! The 
equation of state changes to

$$
\begin{array}{ll}
E \sim S^{6 / 5}\left[\frac{Q_{5}^{3} g_{\mathrm{YM}}^{2} V_{4}}{R}\right]^{-1 / 5}, & p_{\|} \ll E\left(r_{0} \gg q_{1}\right) \\
E \sim S^{3}\left[\frac{g_{\mathrm{YM}}^{2}}{k^{3 / 2} V_{4}^{1 / 4} R}\right] & , \quad p_{\|} \gg E\left(r_{0} \ll q_{1}\right) .
\end{array}
$$

We can understand the first of these in the following way: $g_{\mathrm{YM}}^{2} / R$ in $5+1 \mathrm{~d}$ is the $4+1 \mathrm{~d}$ Yang-Mills coupling, so (1.31) is the same equation of state as the fivebrane gas encountered in section 3.2.2. Thus, at relatively low momenta (entropy dominating over the boost $\alpha_{\text {eff }}^{\prime 1 / 2} p_{\|}$), the Hagedorn transition is one of long, thermally excited effective strings overtaking the gas of modes of the $(2,0)$ supersymmetric tensor field theory that describes the fivebrane dynamics at lower energies.

The second equation of state, Equation (1.32), should describe the 'zero-mode' dynamics of the toroidally compactified fivebrane, if our previous examples serve as an accurate guide. In these single-charge brane systems, we found that the low-entropy structure obeyed infinite momentum frame kinematics of highly boosted objects; indeed, the Hagedorn phase (1.30) exhibits precisely such behavior. To cast (1.32) in this form, we posit that the strongly coupled system occupies an effective transverse four-torus of size

$$
\tilde{V}_{4}=\frac{Q_{5}}{Q_{1}} V_{4}
$$

this is reminiscent of other gauge systems [42][24] where at low entropies the holonomies of the gauge fields arrange themselves in such a way as to modify the box size seen by the effective degrees of freedom. With this redefinition, and the apparent longitudinal box size $\tilde{R}$ arising in the Hagedorn phase, we can recast the localized equations of state (1.31), (1.32) as

$$
\begin{array}{ll}
E \sim S^{6 / 5}\left[k \tilde{V}_{4} \tilde{R}\right]^{-1 / 5}, & p_{\|} \ll E \\
E \sim \frac{1}{p_{\|}}\left[\frac{S^{3 / 2}}{\left(k \tilde{V}_{4}^{1 / 2}\right)^{1 / 2}}\right]^{2}, & p_{\|} \gg E .
\end{array}
$$

Note that these parameter redefinitions uniformly encode the data in all strong-coupling phases. Most importantly, the $Q_{5}^{3}$ count of degrees of freedom in the 'bare' equation of state has been processed into the 'dressed' count of $k=Q_{1} Q_{5}$ degrees of freedom, which is more like what 
we were expecting. Also, equation of state (1.35) now has the form required by infinite momentum frame kinematics, suggesting an object of invariant mass $M \sim S^{3 / 2} /\left(k \tilde{V}_{4}^{1 / 2}\right)^{1 / 2}$ replaces the boosted Hagedorn string below the Hagedorn transtion. This is the equation of state of a $2+1 \mathrm{~d}$ relativistic gas of $k$ degrees of freedom, living in an effective box of size $\tilde{V}_{4}^{1 / 2}$. A good candidate remains to be found for the composition of this effective membrane-like object.

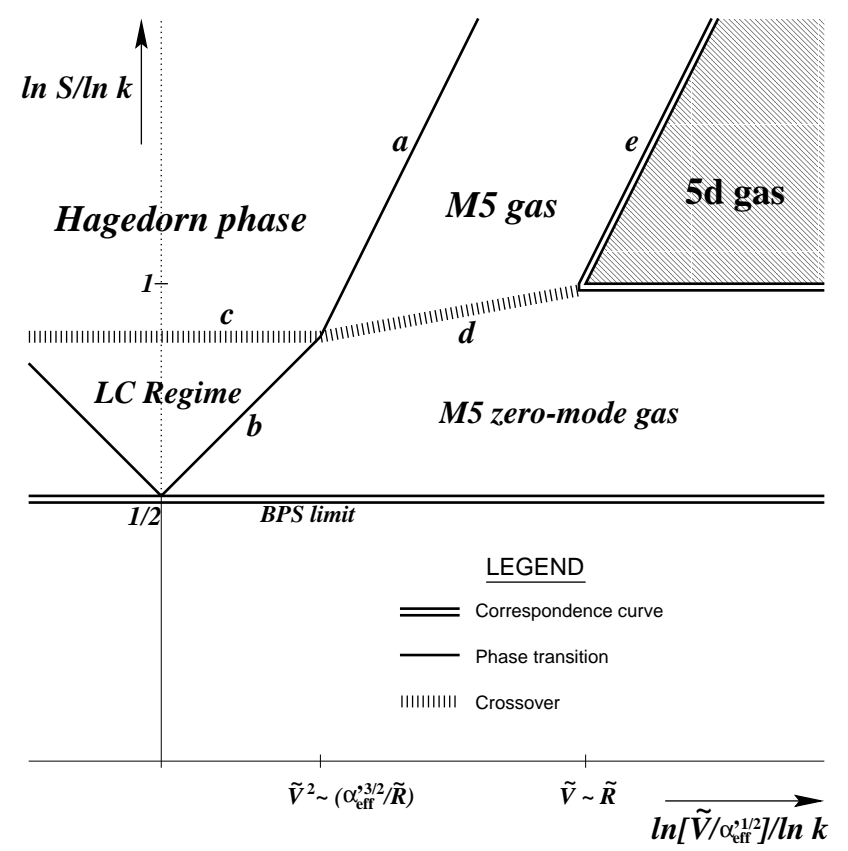

Figure 1.12 Phase diagram of boosted M5-brane 'little strings'.

The phase diagram is plotted in Figure 1.12. The wide dashed line indicates the location of the crossover to infinite momentum frame kinematics at $p_{\|} \sim E$, with the IMF side being that of lower entropy. Further evidence that the reshuffling of the parameters is on the right track comes from the scaling of the various transition curves a-e [7], which are all of the form

$$
\frac{S}{k} \sim\left(\frac{\tilde{R}}{\alpha_{\text {eff }}^{\prime 1 / 2}}\right)^{\alpha}\left(\frac{\tilde{V}_{4}}{\alpha_{\text {eff }}^{\prime 2}}\right)^{\beta},
$$

i.e. the entropy per degree of freedom at the transition is determined entirely by the effective geometry; the expressions in terms of the bare parameters has no such uniform interpretation. 
Remarkably, a system that we otherwise understand very poorly, being some sort of strongly coupled string theory without gravity, has its thermodynamics controlled by black geometry! In particular, the Hagedorn transition of the effective string is a geometrical transition of horizon localization. As in the matrix theory of 11d supergravity, a system whose underlying degrees of freedom are otherwise hard to understand, the introduction of a superselection charge with the interpretation of momentum leads to a simplification. In fact, the analogy is rather direct: The matrix model of $11 \mathrm{~d}$ supergravity describes a system with longitudinal momentum $p_{\|}=N / R_{11}$, in terms of the $N \times N$ matrix quantum mechanics of $N$ D0-branes; here, the matrix model of M5-branes appears to carry boost $p_{\|}=k / \tilde{R}$ and have $k$ degrees of freedom, which might be traced back to the dynamics of the $Q_{1} \times Q_{5}$ matrix of 1-5 strings.

Much remains to be understood, however. The forms of the equation of state (1.35),(1.34) are the low- and high-entropy asymptotics of the full equation of state derived from the D0-D4 black hole localized on a transverse circle, in the limits $r_{0} \ll q_{1}$ and $r_{0} \gg q_{1}$, respectively. One would like to understand the finite size effects that give rise to the membrane-like structure apparent in (1.35); these do not have the structure of a canonical boosting as in (1.30) [7].10 It would be helpful to have a model to explain how the effective box sizes $\tilde{R}$ and $\tilde{V}_{4}$ arise dynamically. For instance, $\tilde{R}$ was motivated as the 'T-dual' of the original winding radius $R$ of the D1-branes, using the effective string scale $\alpha_{\text {eff }}^{\prime}$; however, the little string theory does not really have such a global duality symmetry. The factor of $Q_{5}$ in $\tilde{V}_{4}$ is likely the result of global holonomy of the D5 gauge fields linking together the individual fivebranes as one traverses the cycle of the four-torus; the inverse dependence on $Q_{1}$ seems puzzling, though.

\section{CONCLUDING REMARKS}

It is remarkable that such a wide range of parameter space in the thermodynamics of maximally supersymmetric gauge theory is accessible to study via a set of dual geometrical descriptions. Moreover, the dual geometrical descriptions exemplify in a unifying fashion much of the duality structure of M-theory on tori, linking together the Maldacena and matrix conjectures. Thermodynamic considerations are one of the

\footnotetext{
${ }^{10}$ On the other hand, when the boost is along a direction in which the horizon is smeared, generically the equation of state does not have a canonically boosted form; so we might turn the problem around, and ask why the Hagedorn equation of state (1.30) does have a canonically boosted form. Nevertheless the low-entropy limit of both the Hagedorn equation of state and (1.35) has the standard infinite momentum frame form.
} 
few non-BPS probes we have of strongly couped brane systems, and may (as in section 4) lead us to new insights about their effective degrees of freedom at different scales.

The brane ensemble at finite $\mathrm{N}$ undergoes decay via Hawking radiation; thus the time evolution of the system gradually traces a path on the phase diagram. In our analysis, we have ignored the fact that the brane dynamics on a torus has a large moduli space of flat directions, corresponding to separating the branes transversely; in fact this is perhaps the simplest decay mode for very near-extremal black holes composed out of toroidally wrapped branes. Thermodynamics is valid in the large $\mathrm{N}$ limit, when the decay time can be made arbitrarily long relative to other time scales in the dynamics. But it would seem that we can probe arbitrarily large radii with arbitrarily little energy cost; what happened to the UV/IR relation, Equation (1.13)? It is probe-dependent. Objects probing the flat directions in the moduli space are Dp-branes moving out on the Coulomb branch of the effective $\mathrm{p}+1 \mathrm{~d}$ superYang-Mills theory; the UV/IR relation applies to supergravity modes that do not carry Dpbrane charge, related to single-trace operators in the gauge theory 1112 The lesson is that the softest modes of the system are those that carry Dp-brane charge, and appears to be the key as to when there is a dual representation of quantum gravity in terms of a theory without gravity. The idea is that when we accumulate a high density of charged objects, soft excitations occurring inside the charge radius must carry the charge, or are built from composites of charged objects. Similar phenomena abound in condensed matter systems, the prime example being the Fermi surface of a conductor, where all the light excitations are built out of electronic constituents. The correspondence fails (e.g. for D6-branes on $T^{6}$ ) when there are soft excitations that are not built out of the basic charged constituents; then one loses control over the gravitational degrees of freedom since they are not cut off by the regularity of behavior at high energy in field theory. This breakdown of the correspondence is reflected on the gravity side in the negative specific heat of the corresponding black holes.

\footnotetext{
${ }^{11}$ Matrix theory is the extreme example of this phenomenon - the light excitations are the center-of-mass motions of collections of D0-branes, while the single-trace operators that are perturbations of the supergravity D0-brane geometry require more energy to excite.

${ }^{12}$ In the D3-brane gauge theory on $S^{3}$, the Coulomb branch is lifted by the scalar field coupling to the curvature $\mathcal{R} \phi^{2}$. In this case, the UV/IR relation is more universal.
} 


\section{Acknowledgments}

The material in these lectures reflect the thesis work of my student Vatche Sahakian; I would like to thank him for an enjoyable and stimulating collaboration.

\section{References}

[1] Ofer Aharony, Steven S. Gubser, Juan Maldacena, Hirosi Ooguri, and Yaron Oz. Large $\mathrm{N}$ field theories, string theory and gravity. 1999, hep-th/9905111.

[2] Gary T. Horowitz and Joseph Polchinski. A correspondence principle for black holes and strings. Phys. Rev., D55:6189-6197, 1997, hep-th/9612146.

[3] Juan Maldacena. The large N limit of superconformal field theories and supergravity. Adv. Theor. Math. Phys., 2:231, 1998, hepth/9711200.

[4] L. Susskind and E. Witten. The holographic bound in anti-de Sitter space. 1998, hep-th/9805114.

[5] Emil Martinec and Vatche Sahakian. Black holes and the superYang-Mills phase diagram. II. Phys. Rev., D59:124005, 1999, hep-th/9810224.

[6] Emil Martinec and Vatche Sahakian. Black holes and five-brane thermodynamics. Phys. Rev., D60:064002, 1999, hep-th/9901135.

[7] Emil Martinec and Vatche Sahakian. A note on the thermodynamics of 'little string' theory. 1999, hep-th/9906137.

[8] Vatche Sahakian. Black holes and thermodynamics of nongravitational theories. 1999, hep-th/9906044.

[9] T. Banks, W. Fischler, S. H. Shenker, and L. Susskind. M theory as a matrix model: A conjecture. Phys. Rev., D55:5112-5128, 1997, hep-th/9610043.

[10] T. Banks. Matrix theory. Nucl. Phys. Proc. Suppl., 67:180, 1998, hep-th/9710231.

[11] Nissan Itzhaki, Juan M. Maldacena, Jacob Sonnenschein, and Shimon Yankielowicz. Supergravity and the large N limit of theories with sixteen supercharges. Phys. Rev., D58:046004, 1998, hepth/9802042.

[12] J. L. F. Barbon, I. I. Kogan, and E. Rabinovici. On stringy thresholds in SYM/AdS thermodynamics. Nucl. Phys., B544:104, 1999, hep-th/9809033.

[13] Donam Youm. Black holes and solitons in string theory. Phys. Rept., 316:1, 1999, hep-th/9710046. 
[14] Amanda W. Peet and Joseph Polchinski. UV/IR relations in AdS dynamics. Phys. Rev., D59:065011, 1999, hep-th/9809022.

[15] Robbert Dijkgraaf, Erik Verlinde, and Herman Verlinde. BPS quantization of the five-brane. Nucl. Phys., B486:89-113, 1997, hepth/9604055.

[16] Robbert Dijkgraaf, Erik Verlinde, and Herman Verlinde. 5-d black holes and matrix strings. Nucl. Phys., B506:121, 1997, hepth/9704018.

[17] Nathan Seiberg. New theories in six-dimensions and matrix description of M-theory on $T^{5}$ and $T^{5} / Z_{2}$. Phys. Lett., B408:98-104, 1997, hep-th/9705221.

[18] Amit Giveon, Massimo Porrati, and Eliezer Rabinovici. Target space duality in string theory. Phys. Rept., 244:77-202, 1994, hepth/9401139.

[19] Joseph Polchinski. Tasi lectures on D-branes. 1996, hep-th/9611050.

[20] N. A. Obers and B. Pioline. U-duality and M-theory. 1998, hepth/9809039.

[21] R. Gregory and R. Laflamme. Black strings and $p$-branes are unstable. Phys. Rev. Lett., 70:2837, 1993, hep-th/9301052.

[22] Gary T. Horowitz and Joseph Polchinski. Selfgravitating fundamental strings. Phys. Rev., D57:2557-2563, 1998, hep-th/9707170.

[23] Miao Li, Emil Martinec, and Vatche Sahakian. Black holes and the SYM phase diagram. Phys. Rev., D59:044035, 1999, hepth/9809061.

[24] T. Banks, W. Fischler, I. R. Klebanov, and L. Susskind. Schwarzschild black holes from matrix theory. Phys. Rev. Lett., 80:226-229, 1998, hep-th/9709091.

[25] Gary T. Horowitz and Emil J. Martinec. Comments on black holes in matrix theory. Phys. Rev., D57:4935-4941, 1998, hep-th/9710217.

[26] John H. Schwarz. An SL(2,Z) multiplet of type IIB superstrings. Phys. Lett., B360:13-18, 1995, hep-th/9508143.

[27] Paul S. Aspinwall. Some relationships between dualities in string theory. Nucl. Phys. Proc. Suppl., 46:30, 1996, hep-th/9508154.

[28] Moshe Rozali. Matrix theory and U-duality in seven dimensions. Phys. Lett., B400:260-264, 1997, hep-th/9702136.

[29] Nathan Seiberg. Why is the matrix model correct? Phys. Rev. Lett., 79:3577-3580, 1997, hep-th/9710009.

[30] Lubos Motl. Proposals on nonperturbative superstring interactions. 1997, hep-th/9701025. 
[31] Tom Banks and Nathan Seiberg. Strings from matrices. Nucl. Phys., B497:41-55, 1997, hep-th/9702187.

[32] Robbert Dijkgraaf, Erik Verlinde, and Herman Verlinde. Matrix string theory. Nucl. Phys., B500:43, 1997, hep-th/9703030.

[33] Miao Li and Emil Martinec. Probing matrix black holes. 1998, hep-th/9801070.

[34] Miao Li. Ten-dimensional black hole and the D0-brane threshold bound state. Phys. Rev., D60:066002, 1999, hep-th/9901158.

[35] L. Susskind. Matrix theory black holes and the Gross-Witten transition. 1997, hep-th/9805115.

[36] Nathan Seiberg. Notes on theories with 16 supercharges. Nucl. Phys. Proc. Suppl., 67:158, 1998, hep-th/9705117.

[37] Tom Banks, Michael R. Douglas, Gary T. Horowitz, and Emil Martinec. AdS dynamics from conformal field theory. 1998, hepth/9808016.

[38] Juan M. Maldacena. Black holes and D-branes. Nucl. Phys. Proc. Suppl., 61A:111, 1998, hep-th/9705078.

[39] O. Aharony, M. Berkooz, S. Kachru, N. Seiberg, and E. Silverstein. Matrix description of interacting theories in six dimensions. Adv. Theor. Math. Phys., 1:148-157, 1998, hep-th/9707079.

[40] Michael R. Douglas. Branes within branes. 1995, hep-th/9512077.

[41] Michael R. Douglas. Gauge fields and D-branes. J. Geom. Phys., 28:255, 1998, hep-th/9604198.

[42] Juan M. Maldacena and Leonard Susskind. D-branes and fat black holes. Nucl. Phys., B475:679-690, 1996, hep-th/9604042. 